\title{
Intense geomagnetic storm during Maunder minimum possibly by a quiescent filament eruption
}

\author{
Hiroaki Isobe, Yusuke Ebihara, Akito D. Kawamura, \\ Harufumi Tamazawa, and Hisashi Hayakawa
}

\section{Published version information}

Citation: $\mathrm{H}$ Isobe et al. "Intense geomagnetic storm during Maunder minimum possibly by a quiescent filament eruption." Astrophysical Journal, vol. 887, no. 1 (2019): 7.

DOI: $\underline{10.3847 / 1538-4357 / a b 107 e}$

This version is made available in accordance with publisher policies. Please cite only the published version using the reference above. This is the citation assigned by the publisher at the time of issuing the APV. Please check the publisher's website for any updates. 


\title{
Intense Geomagnetic Storm during Maunder Minimum Possibly by a Quiescent Filament Eruption
}

\author{
Hiroaki Isobe $^{1,2}$ (D) , Yusuke Ebihara ${ }^{2,3}$, Akito D. Kawamura ${ }^{4}$, Harufumi Tamazawa ${ }^{1,5}$, and Hisashi Hayakawa ${ }^{6,7,8}$ (D) \\ ${ }^{1}$ Faculty of Fine Arts, Kyoto City University of Arts Kyoto 610-1197, Japan; hi-isobe@kcua.ac.jp \\ ${ }^{2}$ Unit of Synergetic Studies for Space, Kyoto University Kyoto 606-8502, Japan \\ ${ }^{3}$ Research Institute for Sustainable Humanosphere, Kyoto University, Uji, 611-0011, Japan \\ ${ }^{4}$ Graduate School of Science, Kyoto University, Kyoto 606-8502, Japan \\ ${ }^{5}$ Disaster Prevention Research Institute, Kyoto University, Kyoto 611-0011, Japan \\ ${ }^{6}$ Graduate School of Letters, Osaka University, Toyonaka, 5600043, Japan \\ ${ }^{7}$ Rutherford Appleton Laboratory, Chilton, Didcot, OX11 0QX, UK \\ Received 2019 February 3; revised 2019 March 14; accepted 2019 March 15; published 2019 December 4
}

\begin{abstract}
The Sun occasionally undergoes the so-called grand minima, in which its magnetic activity, measured by the number of sunspots, is suppressed for decades. The most prominent grand minima, since the beginning of telescopic observations of sunspots, is called the Maunder minimum (1645-1715), which occurred when the sunspots became rather scarce. The mechanism underlying the grand minima remains poorly understood as there is little observational information of the solar magnetic field at that time. In this study, we examine the records of one candidate aurora display in China and Japan during the Maunder minimum. The presence of auroras in such mid-magnetic latitudes indicates the occurrence of great geomagnetic storms that are usually produced by strong solar flares. However, the records of contemporary sunspot observations from Europe suggest that, at least for the likely aurora event, there was no large sunspot that could produce a strong flare. Through simple theoretical arguments, we show that this geomagnetic storm could have been generated by an eruption giant quiescent filament or a series of such events.
\end{abstract}

Key words: history and philosophy of astronomy - solar-terrestrial relations - Sun: filaments, prominences - Sun: flares - sunspots

\section{Introduction}

Solar magnetic activity varies with various timescales (Hathaway 2010; Usoskin 2017). Among these are periods called grand minima, in which magnetic activity is significantly suppressed. The Maunder minimum (1645-1715) is the most prominent grand minimum since the beginning of telescopic observations of the Sun (Eddy 1976). The dynamo mechanism of the grand minima is still controversial (Charbonneau 2010), but the states of the solar magnetic field and the heliosphere during the Maunder minimum have been inferred by multiple proxies (Kataoka et al. 2012; Riley et al. 2015; Usoskin et al. 2015). Analyses of cosmogenic radionuclides and sunspot records suggest that the Schwabe cycle persisted even during the Maunder minimum (Ribes \& Nesme-Ribes 1993; Beer et al. 1998; Miyahara et al. 2004; Vaquero et al. 2015). Another useful proxy for past solar activity is the record of the aurora borealis (e.g., Hayakawa et al. 2017a, 2017c, 2019b, 2019d; Stephenson et al. 2019). Quite a few catalogs of the past aurora records were compiled by various authors, as summarized in Chapter 6.5 of Vaquero \& Vázquez (2009).

It should be noted that three proxies of past solar activity, namely, sunspots, cosmogenic isotopes, and mid-latitude auroras, reflect various aspects of the solar magnetic activities. The sunspot number seems to be the most direct indication of a solar magnetic field, but sunspots appear only when a sufficiently large magnetic flux $\left(\gtrsim 10^{19} \mathrm{Mx}\right)$ is concentrated enough to suppress convective energy transport (Leka \& Skumanich 1998). A large part of the solar surface is covered with sparse but significant magnetic flux, which does not appear as a sunspot (de Wijn et al. 2009).

\footnotetext{
${ }^{8}$ JSPS Research Fellow.
}

Cosmogenic radionuclides are created by the precipitation of high-energy particles from space. Their correlation with the solar magnetic field is rather complex (Usoskin 2017). When the Sun is magnetically active, the heliosphere is filled with a strong magnetic field that suppresses the intrusion of galactic cosmic rays into the heliosphere. Thus, the production of cosmogenic isotopes in the terrestrial atmosphere is low. The production rate of radionuclides is also modulated by the secular variation of the geomagnetic field. Moreover, energetic particles produced by intense solar flares/eruptions and resultant interplanetary shocks also precipitate into the Earth's atmosphere and produce radionuclides (Usoskin et al. 2006; Usoskin \& Kovaltsov 2012; Barnard et al. 2018).

The aurora borealis in the mid- to low-latitudinal regions can be regarded as a proxy for large eruptive events in the Sun (Tsurutani et al. 2003; Cliver and Dietrich 2013; Hayakawa et al. 2019c). The physical connections between the solar eruptions and the low-latitude auroras are rather complex, but the widely accepted outline is as follows: Eruptive events in the Sun such as flares and filament eruptions launch coronal mass ejections (CMEs) into interplanetary space. When the southward component of the magnetic field is embedded in shock sheaths and/or the interplanetary CME (ICME), its energy is effectively transferred to the magnetosphere via magnetic reconnection, which enhances the magnetospheric convection, resulting in the development of the ring current and the equatorward expansion of the auroral oval (Akasofu 1963). Empirically, it is known that the latitudinal extent of the aurora oval is correlated with the Dst index, which is a measure of the ring current development (Yokoyama et al. 1998), and the peak Dst index is correlated with the strength of the southward component of the magnetic field and the velocity of the solar wind. Thus, the auroral 
displays in mid- to low-latitude magnetic latitude can be regarded as evidence of strong solar eruptions. However, it should be noted that a strong solar eruption does not cause midto low-latitude aurora when the eruption is not toward the Earth, as in the near-miss extreme ICME in 2012 (Baker et al. 2013), or its magnetic field is not southward, as in the extremely fast ICME episode on 1972 August 4 (Tsurutani et al. 2003).

It is often difficult to confirm whether an aurora-like description in a historical document actually refers to an aurora display or a different phenomenon such as atmospheric optics (e.g., Usoskin et al. 2017; Neuhäuser \& Neuhäuser 2018). Supplementary information, such as time, directions, and the moon phase, is useful in examining the probability that a description is actually a record of an aurora display. Namely, a record is certainly not a record of an aurora display if the display was observed during daytime. Moreover, a record is more likely to be a record of an aurora display if the display was observed in the northern sky (in the case of the Northern Hemisphere) and the moon phase is close to the new moon phase. (Kawamura et al. 2016). Neuhäuser \& Neuhäuser (2015) proposed five criteria for the likeliness of a record to be that of an aurora: color, aurora-typical motion, direction (northward), night-time observations and repetition of the event. While these criteria are apparently useful to evaluate the likeliness of the aurora records, it should be noted that they are not the necessary conditions to be an auroral record and sometimes contradict the actual visual auroral reports in modern observations (Stephenson et al. 2019). For instance, a bright aurora can be seen even in the presence of a bright moon during extremely intense geomagnetic storms such as the great auroral event in 1847 (Cliver \& Dietrich 2013). Likewise, auroral display may be seen equatorward during such intensive magnetic storms. If the equatorial boundary of the aurora oval extended down as low as $\sim 30^{\circ}$ magnetic latitude (MLAT; Green \& Boardsen 2006; Hayakawa et al. 2016, 2017b, 2018a, 2018b, 2019a; Ebihara et al. 2017; Love 2018), the aurora was visible in the equatorward sky from mid-latitude (say, $\sim 50^{\circ}$ ) regions. Stephenson et al. (2019) compared the criteria proposed by Neuhäuser \& Neuhäuser (2015) with actual observational evidence and doubted their validity with considerable counterexamples. After all, simultaneous and independent observations at distant locations provide reasonable support for the interpretation of a display as an aurora because it can exclude the possibility of local phenomena, such as atmospheric scatterings (Wills \& Stephenson 2000).

The aurora records during the Maunder minimum, particularly those from northern to central Europe, have been used to infer the degree of solar magnetic activity during the period (Eddy 1976; Riley et al. 2015; Usoskin et al. 2015). There are also Chinese, Korean, and Japanese records that can be regarded as candidates for auroral displays in these regions (Yau et al. 1995; Xu et al. 2000; Lee et al. 2004; Kawamura et al. 2016), where the geographic latitude $\left(30^{\circ} \sim 40^{\circ}\right)$ is lower than that of central Europe and the magnetic latitude is even lower $\left(20^{\circ} \sim 30^{\circ}\right)$. However, it is uncertain how many of them are the record of true aurora (Usoskin et al. 2017; Neuhäuser \& Neuhäuser 2018).

To the best of our knowledge, the only set of simultaneous, and hence very probable, records of auroral display in East Asia during the Maunder minimum are from 1653 March, as pointed out by Wills \& Stephenson (2000). There is another candidate from 1672, but as discussed in the next section, it was a result of dating error. While there still exist other records that are potentially of aurora display in the mid-latitudes, so far this event is the most likely one during the Maunder minimum. Naturally, the question arises, "how was such a strong geomagnetic storm produced from the Sun in its grand minimum?" In this study, we address this question.

In Section 2, we examine East Asian aurora records and contemporary sunspot records from Europe, and verify that when the 1653 March aurora was seen, the Sun was almost spotless. In Section 3, we show that such a low-latitude aurora can be produced by an eruption of large quiescent filaments, by examining some empirical models. Implications of the study will be discussed in Section 4.

\section{Sunspot and Aurora Records}

\subsection{Simultaneous Aurora Records in East Asia}

Wills \& Stephenson (2000) reported two simultaneous observations of auroral displays on 1653 March 2: one from China and one from Japan. The moon phase of the day was 0.56 . We revisited the original records of these historical reports and provide their translation, observational site, and references as follows.

1. On 1653 March 2, during night, fiery lights illuminated the heaven in four directions. After a while, they became a blue wisp. (Yănzhōufu Cáoxiànzhì v.18, f.10a)

2. On 1653 March 2,... recently, red and white vapors appeared between Nasu and Odawara in Shimotsuke. They were like flags. It is said that the red vapor vanished earlier. (Tokugawa Jikki, v.11, p.70)

The geographic location of the first record, Cáoxiàn, is N34 $59^{\prime}, \mathrm{E} 115^{\circ} 32^{\prime}$. Using the magnetic field model GUFM1 (Jackson et al. 2000), the MLAT is calculated to be N28.4. The location of the observation of the second record from Japan is described as "between Nasu and Odawara in Shimotsuma." Nasu is located at $\mathrm{N} 37^{\circ} 01^{\prime}$, E $140^{\circ} 07^{\prime}$, corresponding to MLAT $=\mathrm{N} 29.9$. Odawara is located at $\mathrm{N} 36^{\circ} 52^{\prime}$, $\mathrm{E} 140^{\circ} 01^{\prime}$, corresponding to MLAT $=\mathrm{N} 29.7$.

In addition to the 1653 March event, an additional candidate, from a set of simultaneous auroral observations in East Asia during the Maunder minimum, can be found in the literature. This candidate is from records of 1672 September. One is a record in Jiāngsū Píngwàngzhì (v.13, f.6b): "On 1672 September 21, during night, crimson vapor filled the heaven like fiery sand." Another candidate can be found in the catalog of astronomical records in Japan, compiled by Osaki (1994), and also in Nakazawa et al. (2004), who presumably referred to Osaki's catalog. According to these publications, there is a record of "Heaven was red" (TEN-AKA-shi in Table 1 of Nakazawa et al. 2004)" on 1672 September 17. However, the contemporary woodprint Honcho-Nendai-ki (v.5, f.42b) dated this record as 1635 September 7. It seems that Osaki (1994) misread the year, as he recorded the year as the 12th year of the Kanbun period, corresponding to 1672 , which was actually the 12 th year of the Kan' ei period (1635) in the original woodprint.

\subsection{Sunspot Records}

While two independent records, dated 1653 March 2, at distant locations are suggestive of an aurora display in such low-latitude regions, we need more independent records to decisively prove this. Furthermore, if these are indeed real aurora records, the question arises as to whether there were 
large sunspots that could produce such geoeffective eruptions. To examine the level of solar activity at the time of the 1653 March 2 event, we consult the contemporary sunspot record by Hevelius (1673), examined by Carrasco et al. (2015). In 1653, there were records of one day in February and seven days in March:

1. February 5 Macula exigua apparuit (small spot appeared).

2. March 1 Nil Macularum (no spots).

3. March 9 Nil Macularum (no spots).

4. March 23 Macula in quadrante Orientali Solis (sunspot in the eastern quadrant of the Sun).

5. March 24 Macula Solis (sunspot).

6. March 25 Binae maculae in Sole (double spots in the Sun).

7. March 27 Iam penitus disparuerant (they had completely disappeared).

8. March 29 Nil Macularum (no spots).

The solar eruption that produced the March 2 aurora should have occurred a day or a few days before. According to Hevelius' record, there was no sunspot on March 1. Does this indicate that the solar eruption that caused the March 2 aurora occurred in the spotless Sun? It is possible that there were sunspots at the end of February, which produced the geoeffective eruption, but they disappeared before Hevelius' observation on March 1 . If the sunspots disappeared from the solar surface within a few days, they must have been very small or almost completely decayed when the eruption occurred.

Another possibility is that the sunspots were very close to the Sun's west limb when they produced the eruption, and they rotated behind the limb before March 1. Many of the extreme geomagnetic storms are produced by eruptions from large active regions (though there are exceptions such as the great aurora on 1909 September 25, see Hayakawa et al. 2019a) that are likely to remain visible one rotation later. The records of sunspots from March 23 to 25 may correspond to this idea, but if the sunspots were close to the west limb at the end of February and remained present one rotation later, they should have appeared in the Western Hemisphere on March 23. This is not consistent with Hevelius' record.

Thus, Hevelius' record suggests that, when the eruption that caused the 1653 March 2 aurora occurred, there were no sunspots present on the solar surface, or only small sunspots existed. However, we note that it is often not straightforward to interpret the historical sunspot records (Muñoz-Jaramillo \& Vaquero 2018), and our argument is of speculative nature.

\section{Physical Interpretations}

\subsection{Intensity of Geomagnetic Storms}

It is known that the equatorward extension of the aurora belt is correlated with the Dst index, a standard measure of the ring current energy. Yokoyama et al. (1998, hereafter Y98) examined 423 geomagnetic storms found that the corrected geomagnetic latitude $\Lambda$ of the equatorward boundary of the auroral oval decreases with the peak Dst index. Y98 assumed that the magnetic field line is the dipole, and that $\Lambda$ is associated with McIlwain's $L$-value (McIlwain 1961) as

$$
L=\frac{1}{\cos ^{2} \Lambda}
$$

Hereinafter, we refer $\Lambda$ to as the magnetic latitude unless otherwise mentioned. In the following, we use the scaling of Y98 to estimate the Dst index of the 1653 March 2 event.

For this purpose, we need to convert the location of the observation site into the magnetic latitude of the equatorward boundary of the aurora. From simple geometry, one can derive the following equation:

$$
(R+h) \cos \left(\Lambda-\Lambda_{0}\right)=R+(R+h) \sin \left(\Lambda-\Lambda_{0}\right) \tan \theta,
$$

where $R$ is the Earth's radius, $h$ is the altitude of the upper boundary of the aurora, $\Lambda_{0}$ is the magnetic latitude of the observational site of the aurora, and $\theta$ is the elevation angle of the aurora. Unfortunately, there is no information on the elevation angle (such as constellations) for this event, so we assume a conservative value of $\theta \sim 10^{\circ}$. Substituting $\Lambda_{0} \sim 29^{\circ}$, a typical altitude of red aurora $h \sim 500 \mathrm{~km}$, and $R \sim 6400 \mathrm{~km}$, we obtain $\Lambda \sim 43^{\circ}$.

In terms of Dst and $\Lambda$, the scaling of Y98 can be defined as

$$
\text { Dst } \approx 12-2200 \cos ^{6} \Lambda(\mathrm{nT})
$$

This value of $\Lambda$ corresponds to Dst $\sim-325 \mathrm{nT}$, which can be regarded as a conservative value because this equation was derived on the basis of 423 geomagnetic storms. Y98 also suggested the other equation as

$$
\text { Dst } \approx 60-3400 \cos ^{6} \Lambda(\mathrm{nT}),
$$

which was derived on the basis of the main phase of the extremely large magnetic storm of 1989 March. With this equation, Dst is estimated to be $\sim-460 \mathrm{nT}$, which may be regarded as the lower limit of Dst. Hereinafter, we take the conservative value of Dst, that is, $-325 \mathrm{nT}$.

\subsection{Possible Solar Origins}

What are the solar origins that are capable of driving Dst $<-300 \mathrm{nT}$ storms in the absence of a large active region? Fast solar winds in corotating interaction regions can drive geomagnetic storms, but they usually do not exceed Dst $=-120 \mathrm{nT}$, and their maximum strength is likely to be $\sim-160$ nT (Richardson et al. 2006).

Another possibility is CMEs from eruptions of quiescent filaments. Strong geomagnetic storms without any association with major flares were recognized as "problem storms" (Dodson \& Hedeman 1964). Later, they were found to be associated with eruptions from the quiet Sun, often accompanied with the disappearance (eruption) of a dark filament (Joselyn \& McIntosh 1981; McAllister et al. 1996; Zhang et al. 2007; Cliver et al. 2009).

McAllister et al. (1996) studied in detail one such geomagnetic storm event that occurred in 1994 April. The event was associated with a giant soft X-ray arcade formation in the quiet Sun, on 1994 April 14, which eventually produced an intense geomagnetic storm (peak Dst of -201 nT) and great aurora on April 17. Although the filament was barely visible, the giant arcade seen in the soft X-ray was considered to be the aftermath of an eruption from a large-scale magnetic neutral line surrounding the polar region of the Sun (Tsuneta et al. 1992; Tripathi et al. 2004).

Figure 1 shows the solar wind parameters (three components of magnetic field, velocity and density) obtained by the IMP8 satellite and the Dst index in 1994 April. Unfortunately, there 


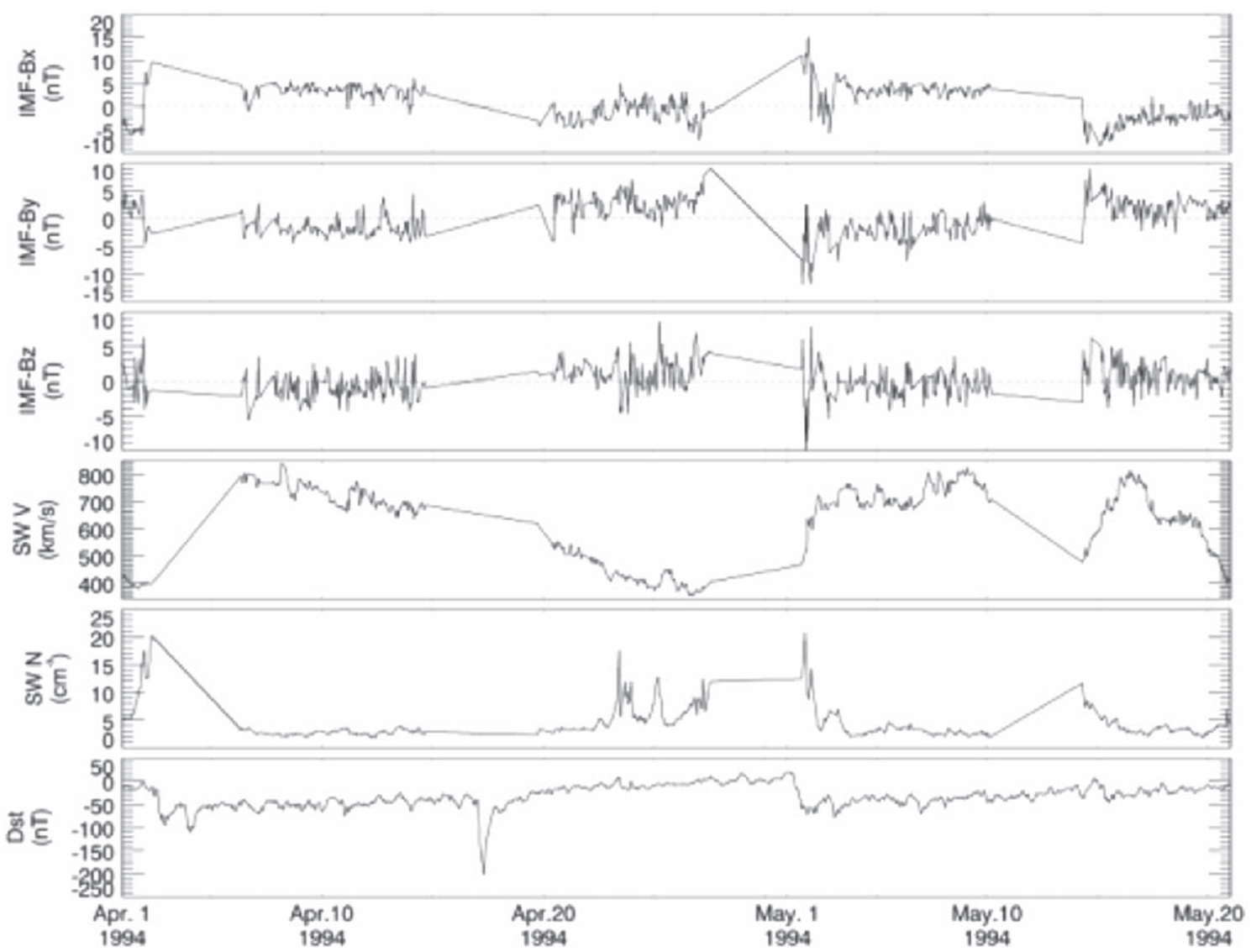

Figure 1. Three components of the magnetic field in the geocentric solar magnetic coordinate, velocity and density in the solar wind measured by IMP8 and the Dst index in 1994 April.

was no record of data during the storm, but one can see that the solar wind before the onset of the storm was relatively fast $\left(\sim 700 \mathrm{~km} \mathrm{~s}^{-1}\right)$. Although one cannot see the ICME in this figure, it was observed by Ulysses at 3 au (McAllister et al. 1996). The average speed of the CME, calculated from the onset times of the X-ray arcade and the geomagnetic storm, is about $600 \mathrm{~km} \mathrm{~s}^{-1}$. An interesting feature, as shown in Figure 1, is that the Dst index was already about $-50 \mathrm{nT}$ before the start of the geomagnetic storm, suggesting a long-lasting moderate activity in the magnetosphere due to the Russell-McPherron effect (Russell \& McPherron 1973). This is evident by the long-lasting toward magnetic field $(B x \gg 0)$ and the date of this event being close to the spring equinox. The high solar wind speed $\left(\sim 700-800 \mathrm{~km} \mathrm{~s}^{-1}\right)$ might participate in the longlasting moderate activity in the solar wind.

During the 1991 November event reported by Cliver et al. (2009), the Dst index of the geomagnetic storm was as low as $-354 \mathrm{nT}$. This is the strongest known geomagnetic storm associated with the eruption of a quiescent filament. Cliver et al. (2009) noted that there was a nearby active region, and hence, the filament may not have arisen from a "pure" quiet Sun. This is possibly related to the relatively high speed $\left(\sim 1000 \mathrm{~km} \mathrm{~s}^{-1}\right)$ of the CME whose origin was a quiescent filament eruption.

In general, CMEs from a quiet Sun are slower. According to a statistical study by Yashiro \& Gopalswamy (2009), the upper limit of the CME speed is well correlated with the peak flux and fluence of soft X-rays, and the speed of the CME associated with the GOES B-class (peak flux lower than $10^{-5} \mathrm{~W} \mathrm{~m}^{-2}$ ) is mostly below $1000 \mathrm{~km} \mathrm{~s}^{-1}$ (see Figure 6 of
Yashiro \& Gopalswamy 2009). On the other hand, the field strength of the magnetic cloud of such slow CMEs can be as large as 30-40 nT (Owens et al. 2005).

Putting these empirical results together, we hypothesize that the 1653 March 2 event was driven by an eruption (or a series of multiple eruptions) from a quiet Sun, which produced an ICME with a relatively slow speed $\left(V \sim 600 \mathrm{~km} \mathrm{~s}^{-1}\right)$ and a strong southward magnetic field $(B \sim 30-40 \mathrm{nT})$.

\subsection{Validity of Solar Wind Parameters}

To quantitatively examine whether such an eruption, as discussed in the previous subsection, can drive a geomagnetic storm as intense as Dst $<-300 \mathrm{nT}$, we consider the empirical evolutionary equation of the Dst index derived by Burton et al. (1975).

$$
\frac{d \mathrm{Dst} *}{d t}=Q(t)-\frac{\mathrm{Dst} *}{\tau_{\text {decay }}} .
$$

Here, $Q(t) \propto V_{\mathrm{SW}} B_{s}$, where $V_{\mathrm{SW}}$ is the solar wind velocity and $B_{s}$ is the southward component of the interplanetary magnetic field, $Q$ is the energy injection rate, and $\tau_{\text {decay }}$ is the decay time.

Note that Dst* is a corrected version of Dst (Burton et al. 1975; O’Brien \& McPherron 2000a) given by

$$
\text { Dst } *=\text { Dst }-b \sqrt{P_{\mathrm{dyn}}}+c,
$$

where $P_{\mathrm{dyn}}$ is the solar wind dynamic pressure, and $b$ and $c$ are constants. Because the second and third terms on the right-hand side are of the order of $10 \mathrm{nT}$, we neglect these terms as they do 


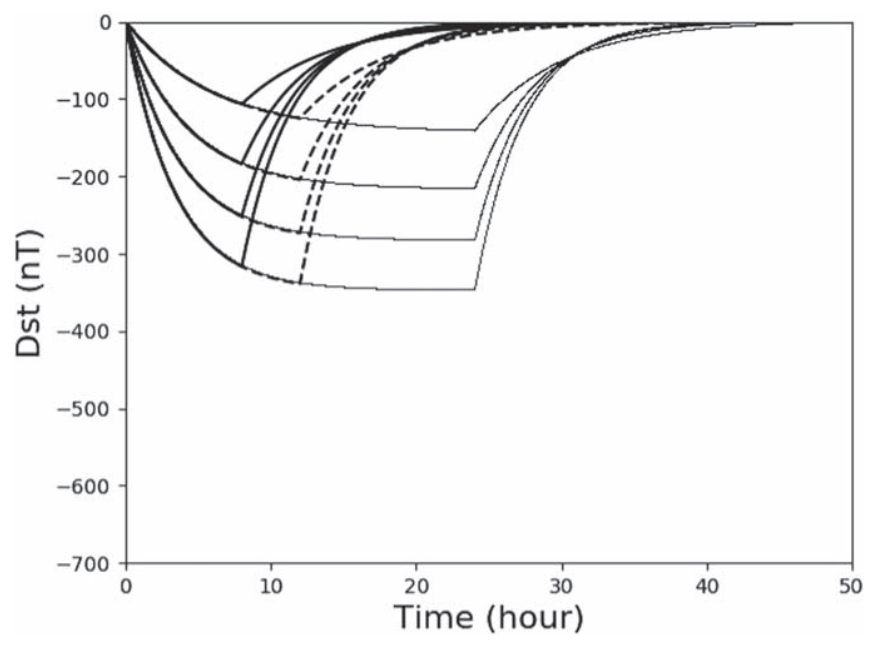

Figure 2. Evolution of Dst index for $V_{\mathrm{SW}}=600 \mathrm{~km} \mathrm{~s}^{-1} ; B_{s}=10,20,30$, $40 \mathrm{nT}, \tau_{\text {dur }}=8,12,24(\mathrm{hr})$, and AK2 model for $\tau_{\text {decay }}$. Thick, dashed, and thin lines correspond to the solutions for $\tau_{\text {dur }}=8,12,24(\mathrm{hr})$, respectively, and the lines with faster decrease in Dst correspond to the solutions for larger $B_{s}$.

not affect the analyses of this study. Therefore, we omit the asterisk throughout the remainder of this paper.

Various models of energy injection rate $Q(t)$ and decay time $\tau_{\text {decay }}$ have been proposed (See review in O'Brien \& McPherron 2000a). O'Brien \& McPherron (2000b) compared different models of $Q(t)$ and $\tau_{\text {decay }}$ and found that the following model (AK2 model) showed the best performance in reproducing the observations.

$$
Q(t)=-4.4\left(V_{\mathrm{SW}} B_{s}-0.5\right)
$$

and

$$
\tau_{\text {decay }}=2.4 \exp \left(\frac{9.74}{4.69+V_{\mathrm{SW}} B_{s}}\right) \text {. }
$$

Here, the electric field $V_{\mathrm{SW}} B_{s}$ is in $\mathrm{mV} \mathrm{m}^{-1}$, and $\tau_{\text {decay }}$ is in hours. Namely, the decay time is shorter for larger energy injections from the solar wind.

When $V_{\mathrm{SW}} B_{s}$ is constant in time, the evolution of Dst stops when the injection and decay terms balance. In an actual situation, $V_{\mathrm{Sw}} B_{s}$ varies with time. For simplicity, we assume that $Q(t)$ is constant during $0 \leqslant t \leqslant \tau_{\text {dur }}$ and set to zero when $\tau_{\text {dur }}<t$.

Because we consider only slow CMEs by quiescent filament eruptions, we fix $V_{\mathrm{SW}}$ to $600 \mathrm{~km} \mathrm{~s}^{-1}$ and change $B_{s}$ and $\tau_{\text {dur. }}$.

Figure 2 shows the numerical solutions of Equation (5) for $B_{s}=10,20,30$, and $40(\mathrm{nT})$ and $\tau_{\mathrm{dur}}$ is 8,12 , and $24(\mathrm{hr})$. $t_{\text {decay }}$ calculated by Equation (8) is 6.0, 4.3, 3.7, and $3.4(\mathrm{hr}$ ) for $B_{s}=10,20,30$, and $40(\mathrm{nT})$, respectively. The dashed, solid, and dotted lines are the solutions when $\tau_{\text {dur }}$ is 8,12 , and 24 (hr), respectively. The solutions with a faster decrease in the Dst correspond to a larger value of $B_{s}$. An obvious feature seen in Figure 2 is that the Dst almost saturates at around $t \sim 10(\mathrm{hr})$ even if the energy injection continues. The saturation is a result of the balance between the energy injection term $Q(t)$ and the decay term Dst $/ \tau_{\text {decay }}$ in Equation (5), thus the saturation value

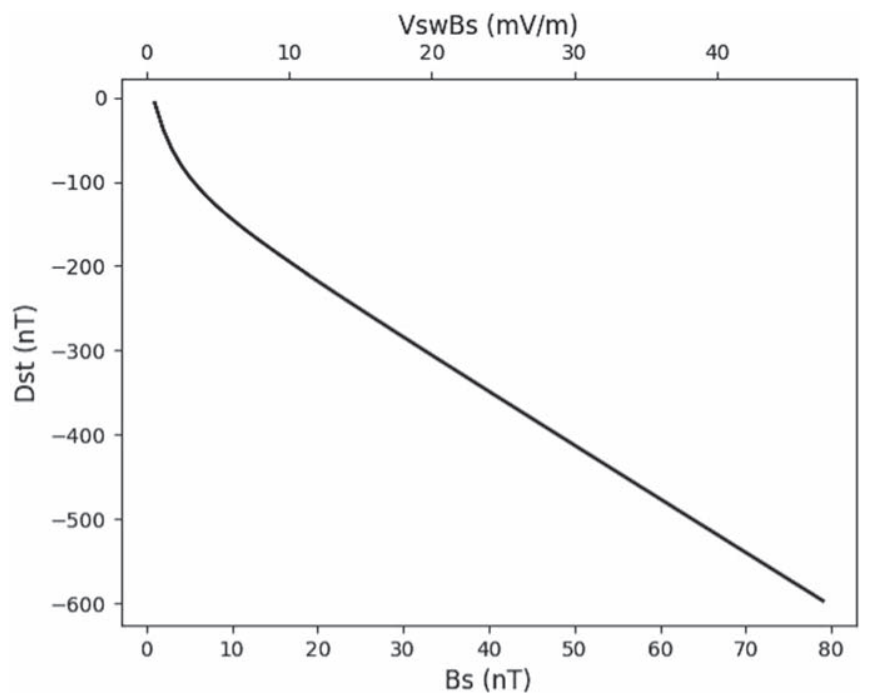

Figure 3. Saturation value of Dst as function of the southward component of solar wind magnetic field $B_{s}$ or associated electric field $V_{\mathrm{SW}} B_{s}$ for the AK2 model. $V_{\mathrm{SW}}=600 \mathrm{~km} \mathrm{~s}^{-1}$ is assumed.

Dst $_{\text {sat }}$ is given by

$$
\begin{aligned}
\operatorname{Dst}_{\mathrm{sat}}= & Q(t) \tau_{\text {decay }}=-10.6\left(V_{\mathrm{SW}} B_{s}-0.5\right) \\
& \times \exp \left(\frac{9.74}{4.69+V_{\mathrm{SW}} B_{s}}\right)
\end{aligned}
$$

and is plotted in Figure 3. From Figures 2 and 3, we conclude that, according to the AK2 model of O'Brien \& McPherron (2000a), the Dst can exceed $-300 \mathrm{nT}$ if $B_{s} \gtrsim 30 \mathrm{nT}$ and $t_{\text {dur }} \gtrsim 8 \mathrm{hr}$.

It should be noted that the AK2 model in O'Brien \& McPherron (2000b) is based on the statistics of moderate (Dst $>-150 \mathrm{nT}$ ) geomagnetic storms, although it reasonably reproduced the Dst evolution of an intense (Dst $<-200 \mathrm{nT}$ ) storm (O'Brien \& McPherron 2000a). Whether the AK2 model (in particular, the form of $\tau_{\text {decay }}$ ) is relevant for extremely intense storms, such as the 1653 March 2 event, is not fully understood, primarily because of the scarcity of such data. Therefore, we also examine the effect of different $t_{\text {decay. }}$. As a reference, in the absence of the decay term, the solution of Equation (5) is a simple linear function of $t$ :

$$
\operatorname{Dst}(t) \approx-830\left(\frac{B_{s}}{40 \mathrm{nT}}\right)\left(\frac{V_{\mathrm{SW}}}{600 \mathrm{~km} \mathrm{~s}^{-1}}\right)\left(\frac{t}{8 \mathrm{hr}}\right)
$$

for $t \leqslant \tau_{\text {dur }}$.

Figure 4 shows the numerical solution of Equation (5) for $B_{s}=40 \mathrm{nT}, \tau_{\text {dur }}=8(\mathrm{hr})$, and $\tau_{\text {decay }}=3,5,7.7,10$, and 20 (hr). One can see that if $\tau_{\text {decay }}$ is sufficiently long, then the peak Dst can reach as low as $-700 \mathrm{nT}$.

Finally, we examine the validity of our hypothesis in terms of the magnetic flux. The total magnetic flux content in an ICME can be estimated from in situ observations by assuming models of the magnetic structure of the ICME (e.g., Dasso et al.

2007). For this study, however, an order-of-magnitude estimate is sufficient. Using the characteristic length $V_{\mathrm{SW}} \tau_{\text {dur }}$ and the magnetic field strength $B_{s}$, the magnetic flux $\Phi$ 


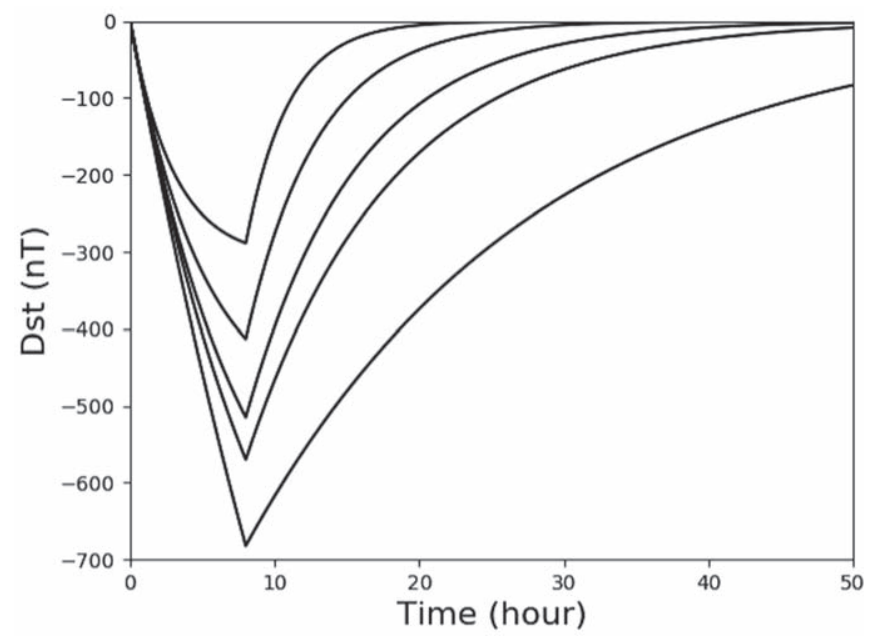

Figure 4. Evolution of Dst index for $B_{s}=40 \mathrm{nT}, \tau_{\text {dur }}=8(\mathrm{hr})$, and $\tau_{\text {decay }}=3$, $5,7.7,10$, and $20(\mathrm{hr})$.

associated with the CME is roughly given by

$$
\begin{aligned}
\Phi \approx & B_{s} V_{\mathrm{SW}}^{2} \tau_{\mathrm{dur}}^{2} \approx 1.2 \times 10^{21}\left(\frac{B_{s}}{40 \mathrm{nT}}\right) \\
& \times\left(\frac{V_{\mathrm{SW}}}{600 \mathrm{~km} \mathrm{~s}^{-1}}\right)^{2}\left(\frac{\tau_{\mathrm{dur}}}{8 \mathrm{hr}}\right)^{2} \mathrm{Mx} .
\end{aligned}
$$

For an average magnetic field strength of $1 \mathrm{G}$, which is typical in the quiet-Sun photosphere, $\Phi=1.2 \times 10^{21} \mathrm{Mx}$ corresponds to an area of $1.2 \times 10^{21} \mathrm{~cm}^{2} \sim 4 \%$ of the solar hemisphere. It is a significant fraction of the solar surface but not an irrelevant value for the magnetic flux associated with a large-scale eruption from a quiet Sun, such as that in 1994 April.

\section{Discussion}

In addition to the sunspot records and cosmogenic radioisotopes, the historical records of aurora displays during the Maunder minimum provide unique and independent information on the solar magnetic activity during this period. To the best of our knowledge, the simultaneous records in China and Japan on 1653 March 2 are the only known information of aurora displays during the Maunder minimum securely confirmed by simultaneous observations. The empirical correlation between the latitude of the aurora and the Dst index indicates that the geomagnetic storm of the 1653 March 2 event was extremely intense (Dst $\lesssim-300 \mathrm{nT}$ ). From contemporary sunspot records and through some simple theoretical arguments, we propose that the Sun was either spotless or with only tiny spots, and that the geomagnetic storm was driven by the eruption of quiescent filament(s).

If the proposed hypothesis is true, its significance is twofold. From the viewpoint of space weather, it indicates that even when the Sun looks very quiet, serious space-weather hazards may occur, although their probability is low. From the viewpoint of solar magnetism, it provides additional constraints on the magnetic configurations in the Sun during the grand minimum.

Here, we discuss additional factors that may enhance the geoeffectiveness of the eruption. The first possible factor is multiple eruptions and CMEs. It has been known that consecutive eruptions can be more geoeffective than an isolated CME (Gopalswamy et al. 2004; Kataoka et al. 2017). Such consecutive CMEs are usually produced by flare-productive active regions (Chatterjee \& Fan 2013). To our knowledge, there are no known intense geomagnetic storms driven by multiple and consecutive eruptions purely from a quiet Sun. However, consecutive eruptions that involve quiescent filaments have also been observed and are known as "sympathetic eruptions" (Schrijver \& Title 2011; Titov et al. 2012).

Another solar factor that may enhance the geoeffectiveness is a fast solar wind from a disk coronal hole. Asai et al. (2009) investigated the active region, NOAA 10798, that emerged in the middle of a disk coronal hole and produced three moderate (M-class) flares. The associated CMEs were very fast $\left(1200 \sim 2400 \mathrm{~km} \mathrm{~s}^{-1}\right)$ because they were in the fast solar wind from the coronal hole. In addition, the faster CME caught up to the preceding, relatively slow $\mathrm{CME}$, and eventually they merged. The combination of fast velocity and the fact that the two consecutive CMEs merged before they arrive at the Earth, enhanced the geoeffectiveness, and consequently an intense geomagnetic storm (Dst $=-216 \mathrm{nT}$ ) occurred. While the assistance of a fast solar wind in the acceleration of CMEs appears to be a feasible mechanism, directly applying the same scenario to quiescent filament eruptions is problematic because it is unlikely that large-scale quiescent filaments exist in a large coronal hole, as a large-scale filament requires a long magnetic neutral line while a large coronal hole requires a large unipolar region.

The last factor we consider is the seasonal effect. It has been known that geomagnetic activity is stronger around the equinoxes than around the solstices, and indeed the 1653 March 2 event occurred not far from the spring equinox. The proposed mechanisms that account for these seasonal variations are the axial hypothesis (Cortie 1912; Bohlin 1977), equinox hypothesis (Bartels 1932; Svalgaard 1977), and RussellMcPherron effect (Russell \& McPherron 1973). The seasonal variation is usually measured by the occurrence rate of the geomagnetic storms or by indices such as $a a$ and Dst indices, and not necessarily by the intensity of the individual storm. However, if the magnetosphere was already disturbed to some extent, it may help to enhance the geomagnetic storm driven by CMEs. The axial hypothesis considers that the Earth is in the heliograhic latitude near the equinoxes, where the solar wind condition can be more hazardous owing to mid-latitude sunspots Cliver et al. (2000) or coronal holes (Bohlin 1977). For the 1653 March 2, event, there was no sunspot, but the effect of the fast solar wind at a higher heliographic latitude may have played a role. The equinox hypothesis attributes the seasonal variation to the varying angle between the Earth's magnetic dipole axis and the Sun-Earth line. According to Cliver et al. (2000), this effect significantly contributes to the seasonal variation but primarily suppresses the coupling efficiency of the solar wind and the magnetosphere near solstices. Thus, it is not relevant as a mechanism for enhancing the geoeffectiveness of eruptions near equinoxes. Finally, in the Russell-McPherron effect, the solar wind magnetic field in a solar equatorial plane has a southward component in the geocentric solar magnetospheric coordinate near the equinoxes. This has been regarded as the principal cause of the enhancement of geomagnetic activity near the equinoxes (Cliver et al. 2000; Zhao \& Zong 2012). However, its contribution to the strongest storms is considered to be 
relatively minor (Lockwood et al. 2016), and it may have played some role in the 1653 May 2 event, too.

We note that although the date was near the spring equinox, there is no direct evidence for the factors discussed above playing any role in the 1653 March 2 event. Future surveys of so-far-unknown records of aurora displays during the Maunder minimum is a promising way to bring new insights on the magnetic activity of the Sun in its grand minima.

This work was supported by Grant-in-Aids from the Ministry of Education, Culture, Sports, Science and Technology of Japan, grant No. JP18H01254 (PI: H. Isobe), JP15H03732 (PI: Y. Ebihara) and a Grant-in-Aid for JSPS Research Fellow JP17J06954 (PI: H. Hayakawa). It was also supported by Kyoto University's Supporting Program for the Interactionbased Initiative Team Studies "Integrated study on human in space" (PI: H. Isobe), the Mission Research Projects of the Research Institute for Sustainable Humanosphere (PI: H. Isobe) and SPIRITS 2017 (PI: Y. Kano) of Kyoto University. H.H. thanks T. Takeda for letting him access Chinese local treatises in the Institute for Research i Humanities of Kyoto University.

\section{ORCID iDs}

Hiroaki Isobe (1) https://orcid.org/0000-0003-2976-5130 Hisashi Hayakawa (ํ) https://orcid.org/0000-0001-5370-3365

\section{References}

Akasofu, S.-I. 1963, JASTP, 26, 1167

Asai, A., Shibata, K., Ishii, T. T., et al. 2009, JGR, 114, A00A21

Baker, D. N., Li, X., Pulkkinen, A., et al. 2013, SpWea, 11, 585

Barnard, L., McCracken, K. G., Owens, M. J., \& Lockwood, M. 2018, JSWSC, 8, A23

Bartels, J. 1932, TeMAE, 37, 1

Beer, J., Tobias, S., \& Weiss, N. 1998, SoPh, 181, 237

Bohlin, J. D. 1977, SoPh, 51, 377

Burton, R. K., McPherron, R. L., \& Russell, C. T. 1975, JGR, 80, 4204

Carrasco, V. M. S., Álvarez, J. V., \& Vaquero, J. M. 2015, SoPh, 290, 2719

Charbonneau, P. 2010, LRSP, 7, 3

Chatterjee, P., \& Fan, Y. 2013, ApJL, 778, L8

Cliver, E. W., Balasubramaniam, K. S., Nitta, N. V., \& Li, X. 2009, JGR, 114, A00A20

Cliver, E. W., \& Dietrich, W. H. 2013, JSWSC, 3, A31

Cliver, E. W., Kamide, Y., \& Ling, A. G. 2000, JGR, 105, 2413

Cortie, A. L. 1912, MNRAS, 73, 52

Dasso, S., Nakwacki, M. S., Démoulin, P., \& Mandrini, C. H. 2007, SoPh, 244,115

de Wijn, A. G., Stenflo, J. O., Solanki, S. K., \& Tsuneta, S. 2009, SSRv, 144, 275

Dodson, H. W., \& Hedeman, E. R. 1964, P\&SS, 12, 393

Ebihara, Y., et al. 2017, SpWea, 15, 1373

Eddy, J. 1976, Sci, 192, 1189

Gopalswamy, N., Yashiro, S., Krucker, S., Stenborg, G., \& Howard, R. A. 2004, JGR, 109, A12105

Green, J. L., \& Boardsen, S. 2006, AdSpR, 38, 130

Hathaway, D. H. 2010, LRSP, 7, 1

Hayakawa, H., Ebihara, Y., Cliver, E. W., et al. 2019a, MNRAS, 484, 4083

Hayakawa, H., Ebihara, Y., Hand, D. P., et al. 2018b, ApJ, 869, 57

Hayakawa, H., Ebihara, Y., Willis, D. M., et al. 2018a, ApJ, 862, 15

Hayawaka, H., Ebihara, Y., Willis, D. M., et al. 2019c, SpWea, in press

Hayakawa, H., Iwahashi, K., Ebihara, Y., et al. 2017b, ApJL, 850, L31

Hayakawa, H., Iwahashi, K., Tamazawa, H., et al. 2016, PASJ, 68, 99
Hayakawa, H., Mitsuma, Y., Ebihara, Y., \& Miyake, F. 2019d, ApJL, 884, L18 Hayakawa, H., Mitsuma, Y., Fujiwara, Y., et al. 2017c, PASJ, 69, 17

Hayakawa, H., Stephenson, F. R., \& Uchikawa, Y. 2019b, SoPh, 294, 42 Hayakawa, H., Tamazawa, H., Uchiyama, Y., et al. 2017a, SoPh, 292, 12 Hevelius, J. 1673, Machina Coelestis. Gdansk.

Jackson, A., Jonkers, A. R. T., \& Walker, M. R. 2000, RSPTA, 358, 957

Joselyn, J. A., \& McIntosh, P. S. 1981, JGR, 86, 4555

Kataoka, R., Isobe, H., Hayakawa, H., et al. 2017, SpWea, 15, 392

Kataoka, R., Miyahara, H., \& Steinhilber, F. 2012, SpWea, 10, S11001

Kawamura, A. D., Hayakawa, H., Tamazawa, H., Miyahara, H., \& Isobe, H. 2016, PASJ, 68, 79

Lee, E. H., Ahn, Y. S., Yang, H. J., \& Chen, K. Y. 2004, SoPh, 224, 373L Leka, K. D., \& Skumanich, A. 1998, ApJ, 507, 454

Lockwood, M., Owens, M. J., Barnard, L. A., et al. 2016, SpWea, 14, 406

Love, J. J. 2018, SpWea, 16, 37

McAllister, A. H., Dryer, M., McIntosh, P., Singer, H., \& Weiss, L. 1996, JGR, 101,13497

McIlwain, C. E. 1961, JGR, 66, 3681

Miyahara, H., Masuda, K., Muraki, Y., et al. 2004, SoPh, 224, 317

Muñoz-Jaramillo, A., \& Vaquero, J. M. 2018, NatAs, 3, 205

Nakazawa, Y., Okada, T., \& Shiokawa, K. 2004, EP\&S, 56, e41

Neuhäuser, R., \& Neuhäuser, D. L. 2015, AN, 336, 930

Neuhäuser, R., \& Neuhäuser, D. L. 2018, EGUGA, 20, 3644

O'Brien, T. P., \& McPherron, R. L. 2000a, JGR, 105, 7707

O’Brien, T. P., \& McPherron, R. L. 2000b, JASTP, 62, 1295

Osaki, S. 1994, A Catalogue of the Early Modern Japanese Astronomical Documents (Kinsei-nihon-tenmon-shiryo) (Tokyo: Hara Shobo) (in Japanese)

Owens, M. J., Cargill, P. J., Pagel, C., Siscoe, G. L., \& Crooker, N. U. 2005, JGR, 110, A01105

Ribes, J. C., \& Nesme-Ribes, E. 1993, A\&A, 276, 549

Richardson, I. G., Webb, D. F., Zhang, J., et al. 2006, JGR, 111, A07S09

Riley, P., Lionello, R., Linker, J. A., et al. 2015, ApJ, 802, 105

Russell, C. T., \& McPherron, R. L. 1973, JGR, 78, 92

Schrijver, C. J., \& Title, A. M. 2011, JGR, 116, A04108

Stephenson, F. R., Willis, D. M., Hayakawa, H., et al. 2019, SoPh, 294, 36

Svalgaard, L. 1977, in Coronal Holes and High Speed Wind Streams, ed. J. B. Zirker (Boulder, CO: Colorado Assoc. Univ. Press), 371

Titov, V. S., Mikic, Z., Török, T., Linker, J. A., \& Panasenco, O. 2012, ApJ, 759, 70

Tripathi, D., Bothmer, V., \& Cremades, H. 2004, A\&A, 422, 337

Tsuneta, S., Takahashi, T., Acton, L. W., et al. 1992, PASJ, 44, L211

Tsurutani, B. T., Gonzalez, W. D., Lakhina, G. S., \& Alex, S. 2003, JGR, 108,1268

Usoskin, I. G. 2017, LRSP, 14, 3

Usoskin, I. G., Arlt, R., Asvestari, E., et al. 2015, A\&A, 581, A95

Usoskin, I. G., \& Kovaltsov, G. A. 2012, ApJ, 757, 92

Usoskin, I. G., Kovaltsov, G. A., Mishina, L. N., Sokoloff, D. D., \& Vaquero, J. 2017, SoPh, 292, 15

Usoskin, I. G., Solanki, S. K., Kovaltsov, G. A., Beer, J., \& Kromer, B. 2006, GeoRL, 33, L08107

Vaquero, J. M., Kovaltsov, G. A., Usoskin, I. G., Carrasco, V. M. S., \& Gallego, M. C. 2015, A\&A, 577, A71

Vaquero, J. M., \& Vázquez, M. 2009, The Sun Recorded Through History: Scientific Data Extracted from Historical Documents (Berlin: Springer)

Wills, D. M., \& Stephenson, F. R. 2000, AnGeo, 18, 1

Xu, Z., Pankenier, D. W., \& Jiang, Y. 2000, East Asian Aechaeoastronomy (Amsterdam: Gordon Breach)

Yashiro, S., \& Gopalswamy, N. 2009, in Proc. IAU Symp. 257, Universal Heliophysical Processes, ed. N. Gopalswamy \& D. F. Webb (Cambridge: Cambridge Univ. Press), 233

Yau, K. K. C., Stepenson, F. R., \& Willis, D. M. 1995, A Catalogue of Auroral Observations from China, Korea and Japan (193 B.C.-A.D. 1770) (Chilton: Rutherford Appleton Lab)

Yokoyama, N., Kamide, Y., \& Miyaoka, H. 1998, AnGeo, 16, 566

Zhang, J., Richardson, I. G., Webb, D. F., et al. 2007, JGR, 112, A10102

Zhao, H., \& Zong, Q.-Z. 2012, JGR, 117, A11222 